\title{
Association of circulating angiotensin converting enzyme activity with respiratory muscle function in infants
}

\author{
Gabriel Dimitriou*1, Despina Papakonstantinou', Eleana F Stavrou², Sotirios Tzifas'1, Aggeliki Vervenioti1, \\ Anny Onufriou ${ }^{3}$, Aglaia Athanassiadou² and Stefanos Mantagos ${ }^{1}$
}

\begin{abstract}
Background: Angiotensin converting enzyme (ACE) gene contains a polymorphism, consisting of either the presence (I) or absence (D) of a 287 base pair fragment. Deletion (D) is associated with increased circulating ACE (CACE) activity. It has been suggested that the D-allele of ACE genotype is associated with power-oriented performance and that CACE activity is correlated with muscle strength. Respiratory muscle function may be similarly influenced. Respiratory muscle strength in infants can be assessed specifically by measurement of the maximum inspiratory pressure during crying $\left(\mathrm{Pi}_{\text {max }}\right)$. Pressure-time index of the respiratory muscles (PTImus) is a non-invasive method, which assesses the load to capacity ratio of the respiratory muscles.
\end{abstract}

The objective of this study was to determine whether increased CACE activity in infants could be related to greater respiratory muscle strength and to investigate the potential association of CACE with PTImus measurements as well as the association of ACE genotypes with CACE activity and respiratory muscle strength in this population.

Methods: Serum ACE activity was assayed by using a UV-kinetic method. ACE genotyping was performed by polymerase chain reaction amplification, using DNA from peripheral blood. PTImus was calculated as $\left(\mathrm{Pi}_{\text {mean }} / \mathrm{Pi}_{\text {max }}\right) \times$ (Ti/Ttot), where $\mathrm{Pi}_{\text {mean }}$ was the mean inspiratory pressure estimated from airway pressure, generated 100 milliseconds after an occlusion $\left(\mathrm{P}_{0.1}\right), \mathrm{Pi}_{\max }$ was the maximum inspiratory pressure and $\mathrm{Ti} / \mathrm{T}$ tot was the ratio of the inspiratory time to the total respiratory cycle time. $\mathrm{Pi}_{\max }$ was the largest pressure generated during brief airway occlusions performed at the end of a spontaneous crying effort.

Results: A hundred and ten infants were studied. Infants with D/D genotype had significantly higher serum ACE activity than infants with I/I or I/D genotypes. CACE activity was significantly related to $\mathrm{Pi}_{\max }$ and inversely related to PTImus. No association between ACE genotypes and Pdi $\mathrm{max}_{\max }$ measurements was found.

Conclusions: These results suggest that a relation in CACE activity and respiratory muscle function may exist in infants. In addition, an association between ACE genotypes and CACE activity, but not respiratory muscle strength, was demonstrated.

\section{Background}

Angiotensin I-converting enzyme (ACE) is a zink metallopeptidase whose main functions are to convert angiotensin I into vasoactive and aldosterone-stimulating peptide angiotensin II and to degrade vasodilator kinins.

* Correspondence: gdimitriou@med.upatras.gr

${ }^{1}$ Neonatal Intensive Care Unit, Department of Pediatrics, University of Patras Medical School, Rio, Patras, Greece

Full list of author information is available at the end of the article
Circulating ACE (cACE) is found in biological fluids and originates from endothelial cells. ACE is also an important component of the local renin-angiotensin systems (RASs), which have been identified in diverse tissues, including lung and skeletal muscles [1,2]. A polymorphism of the human ACE gene has been identified in humans and contains a polymorphism consisting of either the presence (insertion, I) or absence (deletion, D) of a 287 base pair (bp) fragment [3]. The deletion is asso- 
ciated with increased ACE activity in both tissue [4] and circulation [5]. Circulating ACE activity was stable when serially measured in the same individuals, while large differences among subjects were observed [6]. The I/D polymorphism accounts for approximately half of the observed variance in ACE levels [5]. However, the presence of quantitative trait loci controlling ACE levels was suggested [7].

D-allele of ACE genotype has been associated with power-oriented performance, being found in excess in short-distance swimmers [8] and with greater strength gains in the quadriceps muscle [9]. Furthermore, it has been suggested that cACE activity has been associated directly with muscle strength in healthy Caucasians, naïve to strength training [10]. Thus, respiratory muscle function and specific respiratory muscle strength may be similarly influenced.

Respiratory muscle strength in infants can be assessed specifically by measurement of the maximum inspiratory pressure during crying $\left(\mathrm{Pi}_{\max }\right)[11,12]$. Pressure-time index of the respiratory muscles (PTImus) is a non-invasive method, which assess the load to capacity ratio of the respiratory muscles [13]. PTImus has been validated in both adults [14] and infants [15].

The aim of this study was to test the hypothesis that increased cACE activity in infants could be related to greater respiratory muscle strength assessed by measurement of $\mathrm{Pi}_{\max }$. We further investigated the potential association of cACE with PTImus measurements, as well as the association of $\mathrm{ACE}$ genotypes with $\mathrm{CACE}$ activity and respiratory muscle strength in this population.

\section{Methods}

\section{Patients}

Infants cared for at the Neonatal Intensive Care UnitPediatric Department of the University General Hospital of Patras, Greece, were eligible for the study. Infants were entered into the study if parents gave informed written consent. The study was approved by the local Research Ethics Committee. The studied population was recruited from a study examining the association of ACE genotypes on respiratory muscle function in infants. All infants were studied before discharge, in supine position, at least one hour after a feed. Infants had no respiratory symptoms for at least 3 days before measurement. Furthermore, infants were on full oral feeds, had serum electrolytes, calcium, magnesium and phosphates within normal range and did not receive any methylxanthines. Blood sampling for circulating ACE activity determination was performed the previous or the same day of the measurements.

\section{ACE genotype determination}

ACE genotyping was performed on DNA extracted from $0.5 \mathrm{ml}$ of whole blood, collected from an indwelling cath- eter or via peripheral venipuncture during routine blood sampling. The blood samples were stored at $-80^{\circ} \mathrm{C}$ in EDTA vacutainer tubes. The method has been previously described [16]. Briefly, DNA was extracted by using Qiamp spin columns (Blood mini kit- Qiagen, QIAGEN Inc., Germantown, U.S.A). DNA was analyzed by electrophoresis on an agarose gel. DNA amplification of the $16^{\text {th }}$ ACE intron was performed using two sets of primers flanking the polymorphic site, (outer and inner primers), as mistyping of the $\mathrm{D} / \mathrm{D}$ genotype has been reported to occur using conventional amplification with insertion/ deletion (I/D) flanking primer [17].

\section{Plasma ACE activity determination}

An additional $1 \mathrm{ml}$ of whole blood was collected during routine blood sampling. Serum was separated immediately from the whole blood by centrifugation at $1500 \mathrm{~g}$ for $10 \mathrm{~min}$. The samples were stored at $-20^{\circ} \mathrm{C}$ in vacutainer tubes until analysis. Serum ACE activity was assayed by using a UV-kinetic method (Medicon SA) and an AU480 Clinical Chemistry System (Beckman Coulter, Inc, High Wycombe, UK). The determination of ACE was based on the calculation of the rate of absorbance change at 340 $\mathrm{nm}$ during the hydrolysis of the substrate $\mathrm{N}-(3-(2-$ (furyl)acryloyl)-L-phenylalanylglycylglycine (FAPGG) to $\mathrm{N}$-(3-(2-(furyl)acryloyl)-L-phenylalanine (FAP) and glycylglycine. The method has a detection limit of $7 \mathrm{U} / \mathrm{L}$, linearity between 7-140 U/L of ACE and an intra-assay coefficient of variation between $2.26 \%$ and $4.86 \%$.

\section{Measurement of respiratory muscle function}

Airway flow was measured using a pneumotachograph (Mercury F10L, GM Instruments, Kilwinning, Scotland) connected to a differential pressure transducer (DP45, range $\pm 2 \mathrm{~cm} \mathrm{H}_{2} \mathrm{O}$, Validyne Corp, Northridge, CA, USA). Airway pressure (Paw) was measured from a side port on the pneumotachograph, using a differential pressure transducer (DP45, range $\pm 100 \mathrm{~cm} \mathrm{H}_{2} \mathrm{O}$, Validyne Corp, Northridge, CA, USA). The signals from the differential pressure transducers were amplified, using a carrier amplifier (Validyne CD 280, Validyne Corp, Northridge, CA, USA) and they were recorded and displayed in real time on a computer (Dell Optiplex GX620, Dell Inc., Texas, U.S.A) running Labview $^{\mathrm{m}}{ }^{\text {s. }}$ software (National Instruments, Austin, Texas, U.S.A) with analog-to-digital sampling at $100 \mathrm{~Hz}$ (16-bit NI PCI-6036E, National Instruments, Austin, Texas, U.S.A).

\section{Measurement of $\mathrm{Pi}_{\text {max }}$}

To measure $\mathrm{Pi}_{\max }$, a facemask (total deadspace, $4.5 \mathrm{~mL}$ ) was held firmly over the infant's nose and mouth. A small needle leak in the mask was used in order to prevent glottic closure and artificially high $\mathrm{Pi}_{\max }$ [13]. The airway was occluded at the end of a spontaneous crying effort using a unidirectional valve attached to the pneumotachograph, which allowed expiration but not inspiration. The occlu- 
Table 1: Characteristics of the whole study population

\begin{tabular}{|c|c|c|}
\hline Number of studied infants & 110 & \\
\hline Gestational age (weeks) & 36 & $(27-40)$ \\
\hline Birth weight (g) & 2705 & $(960-4150)$ \\
\hline Gender (male) & 62 & $(56.4)$ \\
\hline Preterm & 75 & $(57.7)$ \\
\hline Duration of mechanical ventilation (days) & 0 & $(0-59)$ \\
\hline \multicolumn{3}{|l|}{ Diagnoses on admission } \\
\hline Respiratory distress syndrome (RDS) & 37 & $(33.6)$ \\
\hline Transient tachypnea of the newborn (TTN) & 17 & $(15.5)$ \\
\hline Meconium aspiration syndrome & 5 & $(4.5)$ \\
\hline Infection & 16 & $(14.5)$ \\
\hline Congenital pneumonia & 6 & $(5.5)$ \\
\hline $\begin{array}{l}\text { Prematurity (gestational age } \leq 34 \text { weeks and } \\
\text { birthweight }<2 \mathrm{~kg} \text { without additional } \\
\text { problems on admission) }\end{array}$ & 15 & $(13.7)$ \\
\hline Birth depression & 6 & $(5.5)$ \\
\hline Intrauterine growth retardation & 5 & $(4.5)$ \\
\hline Airleaks & 2 & $(1.8)$ \\
\hline Meconium plug & 1 & $(0.9)$ \\
\hline \multicolumn{3}{|l|}{ At the time of measurement } \\
\hline Postnatal age (days) & 9 & $(1-107)$ \\
\hline Postmenstrual age (PMA) (weeks) & 37.1 & $(32.4-46.7)$ \\
\hline Preterm (PMA<37 weeks) & 57 & $(43.8)$ \\
\hline Weight (g) & 2633 & $(1850-4050)$ \\
\hline CACE activity (U/L) & 42.5 & $(16.7-89.0)$ \\
\hline
\end{tabular}

Table 1: Characteristics of the whole study population

\begin{tabular}{lcc}
\hline $\mathrm{Pi}_{\max }\left(\mathrm{cmH}_{2} \mathrm{O}\right)$ & 65.0 & $(36.9-91.8)$ \\
\hline $\mathrm{Pi}_{\max } /$ weight $\left(\mathrm{cmH}_{2} \mathrm{O} / \mathrm{kg}\right)$ & 24.5 & $(14.5-47.1)$ \\
\hline $\mathrm{PTImus}$ & 0.062 & $(0.023-0.149)$ \\
\hline $\begin{array}{l}\text { Data are demonstrated as n (\%) or median (range). } \\
\text { Abberviations: cACE: circulating ACE; } \mathrm{Pi}_{\text {max }}: \text { maximum inspiratory } \\
\text { pressure during crying; PTImus: pressure-time index of the } \\
\text { respiratory muscles }\end{array}$
\end{tabular}

sion was maintained for at least four inspiratory efforts. At least three sets of airway occlusions were performed and the maximum $\mathrm{Pi}_{\max }$ achieved for individual was recorded.

\section{Measurement of PTImus}

$\mathrm{P}_{0.1}$ was calculated as the airway pressure generated 100 milliseconds after an occlusion, while the infant was quietly breathing. At least four airway occlusions were performed and average $P_{0.1}$ was calculated. Pressure-time index of the inspiratory muscles (PTImus), was calculated as: $\mathrm{PTImus}=\left(\mathrm{Pi}_{\text {mean }} / \mathrm{Pi}_{\text {max }}\right) \times(\mathrm{Ti} / \mathrm{Ttot})$ where $\mathrm{Pi}_{\text {mean }}$ was the average airway pressure during inspiration, obtained from the formula $\mathrm{Pi}_{\text {mean }}=5 \times \mathrm{P}_{0.1} \times \mathrm{Ti}$ [18]. $\mathrm{Pi}_{\text {max }}$ was the maximum inspiratory airway pressure, Ti was the inspiration time and Ttot was the total time for each breath, calculated from the airway flow signal.

Muscle mass increases with maturity and body growth [19] and $\mathrm{P}_{\text {imax }}$ continues to increase outside the neonatal period [11]. Therefore, in order to examine the association of ACE genotype with respiratory muscle strength, $\mathrm{P}_{\text {imax }}$ was also related to body weigth at the time of measurement.

\section{Statistical analysis}

Data was tested for normality using the Shapiro-Wilk and D'Agostino skewness tests. Differences between ACE genotype groups were assessed for statistical significance, using the Kruskal-Wallis and Dunn's post-hoc non parametric and Cramer's V tests, as appropriate. Simple regression analysis was performed to determine whether cACE is related to $\mathrm{Pi}_{\max }$ and PTImus measurements. Stepwise multiple regression analysis was performed to determine if cACE activity is related to respiratory muscle strength, assessed by measurement of $\mathrm{Pi}_{\max }$ and PTImus measurements, independently to weight at measurement, ACE genotyping, postmenstrual age (PMA), gender and support from mechanical ventilation.

Statistical analysis was performed using StatView 5.0 (SAS Institute, Inc., NC, USA) and NCSS 2007 (NCSS, Utah, USA) 


\section{Sample size}

Interim analysis of the data of 50 infants demonstrated a correlation of magnitude $\mathrm{r}=0.23$ between $\mathrm{Pi}_{\max }$ and cACE activity approaching statistical significance. Recruitment of 106 subjects would allow us to detect a correlation of magnitude $\mathrm{r}=0.24$ between $\mathrm{Pi}_{\max }$ and cACE levels with $80 \%$ power at 5\% significance level ("Alpha", the probability of rejecting a true null hypothesis).

\section{Results}

\section{Whole study population}

Between February 2007 and September 2008 one hundred ten infants were recruited. Fifty infants (45.5\%) required ventilation in the initial stage of their illness with a median duration of ventilatory support of 3.3 days (range 1.5-59). The characteristics of the study population are presented in table 1.

Eighteen infants (16.4\%) were homozygous for the Iallele (I/I), 40 (36.4\%) homozygous for the D-allele (D/D) and 52 infants (47.2\%) were heterozygous I/D. ACE genotype distribution was in Hardy-Weinberg equilibrium (HWE), (Chi square for HWE 0.025, $\mathrm{p}=0.874$ ). Overall, there were no significant differences in the characteristics of the infants with I/I, D/D and I/D ACE genotypes (table 2). Neither $\mathrm{Pi}_{\max }$ measurements, nor $\mathrm{Pi}_{\max }$ adjusted for weight at measurement, were statistically different between the three groups, (table 2). Infants with $\mathrm{D} / \mathrm{D}$ genotype had higher serum ACE activity than infants with I/I or I/D genotypes (Kruskal-Wallis, $\mathrm{p}=0.028$; Dunn's test, $\mathrm{z}$-value $=2.37, \mathrm{p}<0.05$ and $\mathrm{z}$-value $=2.12, \mathrm{p}$ $<0.05$, respectively) (table 2), (figure 1 ). No difference, in regards to serum ACE activity, was found between infants with I/I and I/D genotypes (Dunn's test, $\mathrm{z}$-value $=0.83$, n.s). Linear regression analysis demonstrated that $\mathrm{cACE}$ activity was significantly related to $\mathrm{Pi}_{\max }$ after logarithmic transformation $(\mathrm{r}=0.253$, $\mathrm{t}$-value $=2.72, \mathrm{p}=0.0075)$ and inversely related to PTImus $(\mathrm{r}=-0.238$, $\mathrm{t}$-value $=-2.55, \mathrm{p}$ $=0.012$ ). Furthermore, stepwise regression analysis revealed that $\mathrm{Pi}_{\text {max }}$ after logarithmic transformation was significantly related to cACE activity $(p=0.0045)$ and weight at measurement ( $p=0.0081)$, independent of ACE genotyping, PMA, gender and support from mechanical ventilation (table 3 ). In addition, PTImus was related (inversely) to cACE activity $(p=0.00037)$ and to ACE genotypes ( $p=0.00163)$, independent of weight at measurement, PMA, gender and support from mechanical ventilation (table 4).

\section{Infants that never required ventilatory support}

The characteristics of the infants that never required any form of ventilatory support $(n=60)$ are presented in table 5. In this subgroup, 10 infants (16.6\%) were homozygous I/I, 25 (41.7\%) homozygous D/D and 25 infants (41.7\%) were heterozygous I/D (table 6). ACE genotype distribution was in Hardy-Weinberg equilibrium (HWE), (Chi square for HWE 0.741, $\mathrm{p}=0.389$ ).

Infants with I/I, D/D and I/D ACE genotypes, did not differ in regards to their characteristics and in regards to either $\mathrm{Pi}_{\max }$ measurements or $\mathrm{Pi}_{\max }$ adjusted for weight at measurement (table 6). Linear regression analysis demonstrated that cACE activity was significantly related to $\mathrm{Pi}_{\text {max }}$ after logarithmic transformation $(\mathrm{r}=0.421$, $\mathrm{t}$-value $=3.532, \mathrm{p}=0.0008)$ and inversely related to PTImus $(\mathrm{r}=-$ $0.289, \mathrm{t}$-value $=-2.29, \mathrm{p}=0.025)$.

\section{Discussion}

In this study a positive correlation between serum ACE activity and respiratory muscle strength, assessed by $\mathrm{Pi}_{\text {max }}$ measurement, and a negative correlation between serum ACE activity and PTImus in infants, was demonstrated. Infants homozygous for the D-allele had higher cACE activity than infants homozygous for the I-allele and heterozygous I/D. Furthermore, cACE activity was related to $\mathrm{Pi}_{\max }$ and PTImus independent of other factors which could affect respiratory muscle function. The correlation between cACE activity and either $\mathrm{Pi}_{\max }$ or PTImus was replicated on a subpopulation of the main group, consisting of infants that never required any form of respiratory support.

Mouth pressures generated during crying efforts, could provide an index of respiratory muscle strength in awake infants [13]. The test has been previously validated in infants $[11,12] . \mathrm{P}_{\text {imax }}$ measurement is a volitional test of respiratory muscle strength, however, the generated pressures produced during crying, are considered to be maximal [19].

Fatigue of respiratory muscles may result in an inability to maintain adequate alveolar ventilation and respiratory failure. Diaphragmatic pressure-time index (PTIdi) is a measure of the load-capacity ratio of the diaphragm. It describes the pressure-generating capacity of the diaphragm, independent of respiratory frequency or the type of load imposed on the respiratory system [13] and it is closely related to the endurance time, referred to as the point where the inspiratory muscles failed to maintain a task despite maximal effort [20]. The determination of PTIdi, however, is rather invasive, since it requires the placement of an esophageal catheter. Assessment of inspiratory muscle function by measurement of a noninvasive pressure-time index of the respiratory muscles (PTImus) was first described by Gaultier et al. [18]. In spontaneously breathing infants, an agreement between PTImus and PTIdi measurements using Bland and Altman analysis, was found [15].

It has been suggested, based primarily on observational evidence, that the D-allele of ACE polymorphism was 
associated with greater training-related strength gain and power-oriented performance [21]. An excess of the ACE D-allele has been found among elite sprint runners [22] and swimmers [8]. In addition, ACE genotypes in adults, were associated with strength response to muscle training and D-allele carriers experience greater strength increase than II homozygotes [9,23]. ACE genotype, however, is not associated with baseline muscle strength and size [24]. Furthermore, several studies have suggested that I-allele has been associated with superior exercise endurance, being found with increased frequency in elite distance runners [22], rowers [25], triathletes [26] and mountaineers [27]. A study in healthy Caucasian naïve to strength training, suggested that CACE activity was significantly associated with baseline muscle strength [10].

In the current study, ACE genotype was associated with cACE activity, which is in accordance with present literature $[4,5]$. Infants with D/D ACE genotype had increased cACE activity compared to infants either homozygous for the I-allele or heterozygous I/D. Although serum ACE activity was associated with increased respiratory muscle strength, such association was not demonstrated in regards to ACE genotypes. One explanation is that the deletion accounts for approximately $47 \%$ of the intra- individual variation in plasma ACE activity in Caucasians [5]. Furthermore, cACE activity is a continuous variable and would provide greater statistical power than a categorical variable such as ACE genotype. Similar results, however, have been demonstrated by others, where an association between ACE genotyping with pre-training muscle strength was not found $[9,28]$. Nevertheless, the correlation between $\mathrm{Pi}_{\text {max }}$ and cACE activity is rather weak, as approximately only $7 \%$ of the variation in $\mathrm{Pi}_{\max }$ can be accounted for by the variation in cACE activity.

A maturational effect on $\mathrm{P}_{\text {imax }}$ has been previously demonstrated [12]. Several factors could affect $\mathrm{Pi}_{\max }$, such as gestational age, PMA, birthweight and weight at measurement [12]. Muscle mass increases with maturity and body growth [19] and $\mathrm{P}_{\mathrm{imax}}$ continues to increase outside the neonatal period [11]. Furthermore, ACE levels in infants have been reported to be higher than in adults [29], other studies, however, did not show any significant correlation of cACE activity with age [30]. In this study, respiratory muscle assessment was performed at the time of the blood collection, therefore, any maturational effect on $\mathrm{P}_{\text {imax }}$ and variation on cACE activity was avoided. However, to examine the association of ACE genotype

Table 2: Characteristics of infants in relation to ACE genotypes

p value

\begin{tabular}{|c|c|c|c|c|}
\hline ACE genotypes (n) & II (18) & ID (52) & DD (40) & \\
\hline Gestational age (weeks) & $36(27-40)$ & $35(28-40)$ & $36(29-40)$ & $0.680^{\mathrm{a}}$ \\
\hline Birth weight (g) & $2780(960-4150)$ & $2705(1065-3650)$ & $2755(1170-3820)$ & $0.756^{a}$ \\
\hline Male gender & $9(50.0 \%)$ & $28(53.8 \%)$ & $25(62.5 \%)$ & $0.097^{b}$ \\
\hline $\begin{array}{l}\text { Duration of mechanical } \\
\text { ventilation (days) }\end{array}$ & $0(0-59)$ & $2(0-21)$ & $0(0-8)$ & $0.167^{a}$ \\
\hline
\end{tabular}

\begin{tabular}{|c|c|c|c|c|}
\hline \multicolumn{5}{|c|}{ At the time of measurement } \\
\hline Postnatal age (days) & $8.5(1-107)$ & $9.5(1-62)$ & $8(1-54)$ & $0.686^{a}$ \\
\hline Weight (kg) & $2660(1850-4050)$ & $2620(1850-3680)$ & $2657(1880-3540)$ & $0.618^{a}$ \\
\hline cACE activity (U/L) & $39.3(24.3-72.3)$ & $41.1(16.7-71.9)$ & $51.1(23.9-89.0)$ & $0.028^{a}$ \\
\hline $\mathrm{Pi}_{\max }\left(\mathrm{cmH}_{2} \mathrm{O}\right)$ & $71.3(49.3-88.7)$ & $61.1(36.9-90.8)$ & $66.1(42.4-91.8)$ & $0.09^{a}$ \\
\hline $\mathrm{Pi}_{\max } /$ weight $\left(\mathrm{cmH}_{2} \mathrm{O} / \mathrm{kg}\right)$ & $25.3(17.0-37.6)$ & $23.5(14.5-42.3)$ & $24.5(14.9-47.1)$ & $0.267^{a}$ \\
\hline
\end{tabular}

Data are demonstrated as $\mathrm{n}$ (\%) or median (range).

aKruskal-Wallis test ${ }^{\mathrm{b}}$ Cramer's $\mathrm{V}$ test 
Table 3: Results of stepwise regression analysis of factors related to $\mathrm{Pi}_{\text {max }}$ measurements

\begin{tabular}{|c|c|c|c|}
\hline & Partial Correlation Coefficient & t Value & Significance \\
\hline \multicolumn{4}{|c|}{ Dependent variable: $\mathrm{Pi}_{\max }$} \\
\hline CACE activity & 0.262 & 2.899 & 0.0045 \\
\hline Weight at measurement & 0.244 & 2.697 & 0.0081 \\
\hline ACE genotypes & & 0.317 & 0.752 \\
\hline Postmenstrual age & & 0.283 & 0.778 \\
\hline Gender & & 0.077 & 0.939 \\
\hline Duration of mechanicalve & & 1.451 & 0.150 \\
\hline
\end{tabular}

with respiratory muscle strength, $\mathrm{P}_{\text {imax }}$ was also related to body weigth at the time of measurement.

The primary aim of this study was to examine the association of cACE activity with respiratory muscle strength in infants. Secondary aims were to investigate the potential association of $\mathrm{CACE}$ with PTImus measurements and ACE genotypes with cACE activity and respiratory muscle strength in this population. An association between ACE genotypes and PTImus in infants has been previously shown [31]. Thus, this issue was not examined in this study. However, ACE genotype was included in the stepwise regression analysis, as it is now known that it is strongly correlated with PTImus.

The association of cACE activity and respiratory muscle strength may be mediated through synthesis of angio- tensin II (Ang II). Ang II could possibly act as a growth factor in cardiac muscle [32] and its effect may be mediated through Ang II type 1 (AT1) receptor [32]. Furthermore, Ang II may be necessary for optimal overloadinduced skeletal muscle hypertrophy, acting at least in part via an AT(1) receptor-dependent pathway [33]. The physiological properties of a motor unit correlate with the histochemical properties of the constituent muscle fibres [34]. ACE D-allele compared to I-allele is associated with an increased percentage of fast-twitch type IIb skeletal muscle fibres [35], which produce greater force per unit of cross-sectional area [36]. Ang II may be also important in the redirection of blood flow from type I, fatigue-resistant, to type II, fast-twitch, muscle fibres [37]. Furthermore, in animal studies, Ang II infused into

Table 4: Results of stepwise regression analysis of factors related to PTImus measurements

\begin{tabular}{|c|c|c|c|}
\hline & Partial Correlation Coefficient & t Value & Significance \\
\hline \multicolumn{4}{|l|}{ Dependent variable: PTImus } \\
\hline cACE activity & -0.339 & -3.675 & 0.00037 \\
\hline ACE genotypes & 0.298 & 3.233 & 0.00163 \\
\hline Weight at measurement & & 1.944 & 0.055 \\
\hline Postmenstrual age & & 0.792 & 0.430 \\
\hline Gender & & 1.364 & 0.169 \\
\hline Duration of mechanical ventilation & & 0.490 & 0.625 \\
\hline
\end{tabular}




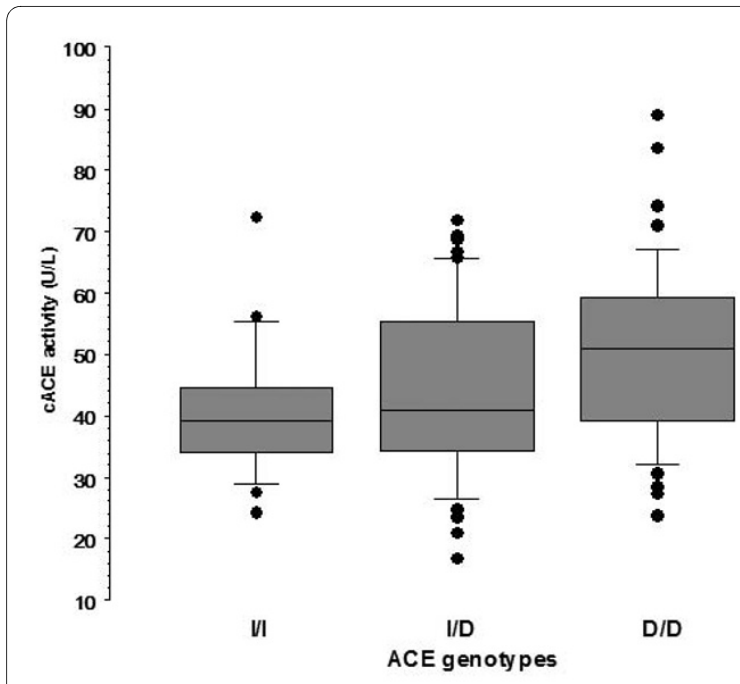

Figure 1 Comparison of CACE activity results in relation with ACE genotypes. Comparison of CACE activity results of infants with $\mathrm{I} / \mathrm{I}(\mathrm{n}=$ 18), I/D ( $n=52)$ and $D / D(n=40)$ ACE genotypes. Box and whisker plot: fine horizontal lines represent $10^{\text {th }}, 25^{\text {th }}, 50^{\text {th }}, 75^{\text {th }}$ and $90^{\text {th }}$ centiles of CACE. Outliers are plotted as discrete data points.

rat hindlimps increases the tension during tetanic stimulation [37]. Other actions of Ang II, that might explain the association between CACE activity and respiratory muscle strength, include the increased noradrenaline release from peripheral sympathetic nerve terminals and the CNS, facilitating sympathetic transmission [38,39]. Circulating ACE may also influence diaphragmatic muscle strength, through the degradation of kinins. In animal studies, bradykinin reduces the phenylephrine-induced hypertrophy of cardiomyocytes [40]. Thus, elevated cACE may influence muscle strength via this pathway.

Several factors may affect respiratory muscle function, such as nutrition [41], prolonged ventilatory support [42], drugs [43-45], as well as phosphate [46], calcium [47] and magnesium [48] blood levels. In addition, hypoxia [49] and hypercapnia [50] reduce diaphragmatic contractility in young piglets. All infants were measured prior to discharge, being free of any respiratory symptoms and on full enteral feeds, they did not receive any medication and their biochemistry blood tests were within the normal range during measurements.

This study has potentially important implications, given the availability of ACE inhibitors. A recent study has demonstrated that in patients with chronic heart failure, long-term therapy with ACE inhibitors improved respiratory muscle strength [51]. However, it was an uncontrolled observational study with a very small sample size. Maximum inspiratory pressure measurement is a volitional test, therefore, in order to assess respiratory muscle strength in subjects with chronic heart failure under therapy, other factors that would interfere with
Table 5: Characteristics of infants that never required respiratory support

\begin{tabular}{|c|c|c|}
\hline Number of studied infants & 60 & \\
\hline Gestational age (weeks) & 37 & $(30-40)$ \\
\hline Birth weight (g) & 2845 & $(1260-3820)$ \\
\hline Gender (male) & 36 & $(60.0)$ \\
\hline Preterm & 28 & $(46.7)$ \\
\hline \multicolumn{3}{|l|}{ At the time of measurement } \\
\hline Postnatal age (days) & 6.5 & $(1-31)$ \\
\hline Postmenstrual age (PMA) (weeks) & 37.6 & $(32.7-41.4)$ \\
\hline Preterm (PMA<37 weeks) & 22 & $(36.7)$ \\
\hline Weight (g) & 2765 & $(1880-3680)$ \\
\hline CACE activity (U/L) & 44.3 & $(16.7-89.0)$ \\
\hline $\mathrm{Pi}_{\max }\left(\mathrm{cmH}_{2} \mathrm{O}\right)$ & 64.3 & $(36.9-91.8)$ \\
\hline $\mathrm{Pi}_{\max } /$ weight $\left(\mathrm{cmH}_{2} \mathrm{O} / \mathrm{kg}\right)$ & 23.9 & $(14.5-47.1)$ \\
\hline PTImus & 0.061 & $(0.028-0.117)$ \\
\hline
\end{tabular}

Data are demonstrated as $\mathrm{n}(\%)$ or median (range).

respiratory muscle function, should be taken into account. Some studies have demonstrated that ACE inhibitor treatment improves exercise capacity $[52,53]$ and decrease long term decline in physical function in elderly adults [54]. However, all studies were observational and referred to either disable, hypertensive subjects or adults with congestive heart failure.

\section{Conclusions}

These results suggest that a relation in cACE activity and respiratory muscle function, as assessed by measurement of $\mathrm{Pi}_{\max }$ and PTImus, may exist in infants. No association between $\mathrm{ACE}$ genotypes and $\mathrm{Pi}_{\max }$ measurements was found. In addition, an association of D-allele of ACE genotype with increased cACE activity in infants was demonstrated. Circulating ACE accounts for only a small proportion of the total body RAS, therefore, ACE activity in muscles may be a more important factor in regards to respiratory muscle properties. Further work is required 
Table 6: Characteristics of infants that never required respiratory support in relation to ACE genotypes

p value

\begin{tabular}{|c|c|c|c|c|}
\hline ACE genotypes (n) & II (10) & ID (25) & DD (25) & \\
\hline Gestational age(weeks) & $36(32-40)$ & $37(30-40)$ & $37(31-40)$ & $0.992^{\mathrm{a}}$ \\
\hline Birth weight (g) & $2780(1350-3650)$ & $2820(1500-3650)$ & $2850(1260-3820)$ & $0.924^{a}$ \\
\hline Male gender & $6(60.0 \%)$ & $14(56.0 \%)$ & $16(64.0 \%)$ & $0.075^{b}$ \\
\hline \multicolumn{5}{|c|}{ At the time of measurement } \\
\hline Postnatal age (days) & $7(1-26)$ & $6(1-30)$ & $8(1-31)$ & $0.834^{a}$ \\
\hline Weight (kg) & $2675(1950-3550)$ & $2680(1950-3680)$ & $2830(1880-3540)$ & $0.951^{\mathrm{a}}$ \\
\hline CACE activity (U/L) & $40.2(24.3-52.8)$ & $41.4(16.7-66.8)$ & $51.8(27.4-89.0)$ & $0.046^{a}$ \\
\hline $\mathrm{Pi}_{\max }\left(\mathrm{cmH}_{2} \mathrm{O}\right)$ & $73.8(49.3-88.7)$ & $61.1(36.9-88.4)$ & $68.1(47.4-91.8)$ & $0.182^{\mathrm{a}}$ \\
\hline $\mathrm{Pi}_{\max } /$ weight $\left(\mathrm{cmH}_{2} \mathrm{O} / \mathrm{kg}\right)$ & $24.9(17.0-37.6)$ & $22.5(14.5-42.3)$ & $24.3(14.9-47.1)$ & $0.347^{a}$ \\
\hline
\end{tabular}

Data are demonstrated as $\mathrm{n}$ (\%) or median (range).

aKruskal-Wallis test ${ }^{\text {b }}$ Cramer's $V$ test

to clarify the effect of ACE inhibitor treatment on respiratory muscle function.

\section{Abbreviations}

ACE: angiotensin converting enzyme; Ang II: angiotensin II; CACE: circulating ACE; HWE: Hardy-Weinberg equilibrium; $\mathrm{Pi}_{\text {max }}$ : maximum inspiratory pressure during crying; PMA: postmenstrual age; PTIdi: diaphragmatic pressure-time index; PTImus: pressure-time index of the respiratory muscles; RAS: reninangiotensin system;

\section{Competing interests}

The authors declare that they have no competing interests.

\section{Authors' contributions}

GD, AA and SM conceived the study; DP and ST collected the subjects' samples and performed the study clinical measurements; DP and EFS performed the genetic studies; EFS and AO carried out the assays for CACE activity determination; AV collected subjects' clinical information; GD, DP and AV performed the data analysis and statistical analysis; GD and DP wrote the first draft of the paper to which all authors subsequently made contributions. All authors read and approved the final manuscript.

\section{Author Details}

${ }^{1}$ Neonatal Intensive Care Unit, Department of Pediatrics, University of Patras Medical School, Rio, Patras, Greece, 2Department of General Biology, University of Patras Medical School, Rio, Patras, Greece and 'BDepartment of Biochemistry, University Hospital of Patras, Rio, Patras, Greece

Received: 17 February 2010 Accepted: 12 May 2010

Published: 12 May 2010

\section{References}

1. Woods DR, Pollard AJ, Collier DJ, Jamshidi Y, Vassiliou V, Hawe E, Humphries SE, Montgomery HE: Insertion/deletion polymorphism of the angiotensin I-converting enzyme gene and arterial oxygen saturation at high altitude. Am J Respir Crit Care Med 2002, 166(3):362-366

2. Jones A, Woods DR: Skeletal muscle RAS and exercise performance. Int J Biochem Cell Biol 2003, 35(6):855-866.

3. Tiret L, Rigat B, Visvikis S, Breda C, Corvol P, Cambien F, Soubrier F: Evidence, from combined segregation and linkage analysis, that a variant of the angiotensin I-converting enzyme (ACE) gene controls plasma ACE levels. Am J Hum Genet 1992, 51(1):197-205.

4. Danser AH, Schalekamp MA, Bax WA, Brink AM van den, Saxena PR, Riegger GA, Schunkert $\mathrm{H}$ : Angiotensin-converting enzyme in the human heart. Effect of the deletion/insertion polymorphism. Circulation 1995, 92(6):1387-1388.

5. Rigat B, Hubert C, Alhenc-Gelas F, Cambien F, Corvol P, Soubrier F: An insertion/deletion polymorphism in the angiotensin l-converting enzyme gene accounting for half the variance of serum enzyme levels. J Clin Invest 1990, 86(4):1343-1346.

6. Alhenc-Gelas F, Richard J, Courbon D, Warnet JM, Corvol P: Distribution of plasma angiotensin I-converting enzyme levels in healthy men: relationship to environmental and hormonal parameters. $J \mathrm{Lab} C \mathrm{Clin}$ Med 1991, 117(1):33-39.

7. Villard E, Tiret L, Visvikis S, Rakotovao R, Cambien F, Soubrier F: Identification of new polymorphisms of the angiotensin l-converting enzyme (ACE) gene, and study of their relationship to plasma ACE levels by two-QTL segregation-linkage analysis. Am J Hum Genet 1996, 58(6):1268-1278.

8. Woods D, Hickman M, Jamshidi Y, Brull D, Vassiliou V, Jones A, Humphries S, Montgomery H: Elite swimmers and the D allele of the ACE I/D polymorphism. Hum Genet 2001, 108(3):230-232.

9. Folland J, Leach B, Little T, Hawker K, Myerson S, Montgomery H, Jones D: Angiotensin-converting enzyme genotype affects the response of human skeletal muscle to functional overload. Exp Physiol 2000 85(5):575-579

10. Williams AG, Day SH, Folland JP, Gohlke P, Dhamrait S, Montgomery HE: Circulating angiotensin converting enzyme activity is correlated with muscle strength. Med Sci Sports Exerc 2005, 37(6):944-948. 
11. Shardonofsky FR, Perez-Chada D, Carmuega E, Milic-Emili J: Airway pressures during crying in healthy infants. Pediatr Pulmonol 1989, 6(1):14-18.

12. Dimitriou G, Greenoug A, Dyke H, Rafferty GF: Maximal airway pressures during crying in healthy preterm and term neonates. Early Hum Dev 2000, 57(2):149-156.

13. ATS/ERS Statement on respiratory muscle testing. Am J Respir Crit Care Med 2002, 166(4):518-624

14. Ramonatxo M, Boulard P, Prefaut C: Validation of a noninvasive tensiontime index of inspiratory muscles. J Appl Physio/ 1995, 78(2):646-653.

15. Dimitriou G, Patel S, Patel J, Rafferty GF, Greenough A: A non-invasive tension-time index of respiratory muscles in neonates [abstract]. Eur Respir Journal 2004, 24(suppl 48):233s.

16. Lindpaintner K, Pfeffer MA, Kreutz R, Stampfer MJ, Grodstein F, LaMotte F Buring J, Hennekens $\mathrm{CH}$ : A prospective evaluation of an angiotensinconverting-enzyme gene polymorphism and the risk of ischemic heart disease. NEng/ J Med 1995, 332(11):706-711

17. Shanmugam V, Sell KW, Saha BK: Mistyping ACE heterozygotes. $P C R$ Methods App/ 1993, 3(2):120-121.

18. Gaultier C, Boule M, Tournier G, Girard F: Inspiratory force reserve of the respiratory muscles in children with chronic obstructive pulmonary disease. Am Rev Respir Dis 1985, 131(6):811-815.

19. Scott CB, Nickerson BG, Sargent CW, Platzker AC, Warburton D, Keens TG Developmental pattern of maximal transdiaphragmatic pressure in infants during crying. Pediatr Res 1983, 17(9):707-709.

20. Bellemare F, Grassino A: Effect of pressure and timing of contraction on human diaphragm fatigue. J Appl Physiol 1982, 53(5):1 190-1195.

21. Jones A, Montgomery HE, Woods DR: Human performance: a role for the ACE genotype? Exerc Sport Sci Rev 2002, 30(4):184-190.

22. Myerson S, Hemingway H, Budget R, Martin J, Humphries S, Montgomery $\mathrm{H}$ : Human angiotensin I-converting enzyme gene and endurance performance. J Appl Physio/ 1999, 87(4):1313-1316.

23. Giaccaglia V, Nicklas B, Kritchevsky S, Mychalecky J, Messier S, Bleecker E, Pahor M: Interaction between angiotensin converting enzyme insertion/deletion genotype and exercise training on knee extensor strength in older individuals. Int J Sports Med 2008, 29(1):40-44.

24. Pescatello LS, Kostek MA, Gordish-Dressman H, Thompson PD, Seip RL, Price TB, Angelopoulos TJ, Clarkson PM, Gordon PM, Moyna NM, et al:: ACE ID genotype and the muscle strength and size response to unilateral resistance training. Med Sci Sports Exerc 2006, 38(6):1074-1081.

25. Gayagay G, Yu B, Hambly B, Boston T, Hahn A, Celermajer DS, Trent RJ: Elite endurance athletes and the ACE I allele--the role of genes in athletic performance. Hum Genet 1998, 103(1):48-50.

26. Collins M, Xenophontos SL, Cariolou MA, Mokone GG, Hudson DE, Anastasiades L, Noakes TD: The ACE gene and endurance performance during the South African Ironman Triathlons. Med Sci Sports Exerc 2004, 36(8):1314-1320.

27. Montgomery HE, Marshall R, Hemingway H, Myerson S, Clarkson P, Dollery C, Hayward M, Holliman DE, Jubb M, World M, et al:: Human gene for physical performance. Nature 1998, 393(6682):221-222.

28. Thomis MA, Huygens W, Heuninckx S, Chagnon M, Maes HH, Claessens AL, Vlietinck R, Bouchard C, Beunen GP: Exploration of myostatin polymorphisms and the angiotensin-converting enzyme insertion/ deletion genotype in responses of human muscle to strength training. Eur J Appl Physio/ 2004, 92(3):267-274.

29. Rohrbach MS, DeRemee RA: Age dependence of serum angiotensinconverting enzyme activity. Lancet 1979, 2(8135):196

30. Fiselier TJ, Lijnen P, Monnens $L$, van Munster $P$, Jansen M, Peer P: Levels of renin, angiotensin I and II, angiotensin-converting enzyme and aldosterone in infancy and childhood. Eur J Pediatr 1983, 141(1):3-7.

31. Papakonstantinou D, Stavrou E, Tzifas S, Mermiga A, Athanassiadou A, Mantagos S, Dimitriou G: Effect of angiotensin converting enzyme (ACE) gene polymorphism on endurance of respiratory muscles in infants [abstract]. Early Hum Dev 2008, 84(suppl):S89-90

32. Dostal DE, Baker KM: Angiotensin II stimulation of left ventricular hypertrophy in adult rat heart. Mediation by the AT1 receptor. Am J Hypertens 1992, $5(5$ Pt 1):276-280.

33. Gordon SE, Davis BS, Carlson CJ, Booth FW: ANG II is required for optimal overload-induced skeletal muscle hypertrophy. Am J Physiol Endocrinol Metab 2001, 280(1):E150-159.

34. Sieck GC: Diaphragm muscle: structural and functional organization. Clin Chest Med 1988, 9(2):195-210.
35. Zhang B, Tanaka H, Shono N, Miura S, Kiyonaga A, Shindo M, Saku K: The I allele of the angiotensin-converting enzyme gene is associated with an increased percentage of slow-twitch type I fibers in human skeletal muscle. Clin Genet 2003, 63(2):139-144.

36. Bottinelli R, Canepari M, Pellegrino MA, Reggiani C: Force-velocity properties of human skeletal muscle fibres: myosin heavy chain isoform and temperature dependence. J Physiol 1996, 495(Pt 2):573-586.

37. Rattigan S, Dora KA, Tong AC, Clark MG: Perfused skeletal muscle contraction and metabolism improved by angiotensin II-mediated vasoconstriction. Am J Physiol 1996, 271(1 Pt 1):E96-103.

38. Saxena PR: Interaction between the renin-angiotensin-aldosterone and sympathetic nervous systems. J Cardiovasc Pharmacol 1992, 19(Suppl 6): $580-88$

39. Story DF, Ziogas J: Interaction of angiotensin with noradrenergic neuroeffector transmission. Trends Pharmacol Sci 1987, 8(7):269-271.

40. Ishigai Y, Mori T, Ikeda T, Fukuzawa A, Shibano T: Role of bradykinin-NO pathway in prevention of cardiac hypertrophy by ACE inhibitor in rat cardiomyocytes. Am J Physiol 1997, 273(6 Pt 2):H2659-2663.

41. Arora NS, Rochester DF: Effect of body weight and muscularity on human diaphragm muscle mass, thickness, and area. J Appl Physiol 1982, 52(1):64-70

42. Tobin MJ, Alex C: Discontinuation of mechanical ventilation. In Principles and Practice of Mechanical Ventilation Edited by: Tobin M. New York: McGraw-Hill; 1994:1177-1206.

43. Aubier M, Murciano D, Menu Y, Boczkowski J, Mal H, Pariente R: Dopamine effects on diaphragmatic strength during acute respiratory failure in chronic obstructive pulmonary disease. Ann Intern Med 1989, 110(1):17-23.

44. Vires N, Aubier M, Murciano D, Fleury B, Talamo C, Pariente R: Effects of aminophylline on diaphragmatic fatigue during acute respiratory failure. Am Rev Respir Dis 1984, 129(3):396-402.

45. Supinski GS, Levin S, Kelsen SG: Caffeine effect on respiratory muscle endurance and sense of effort during loaded breathing. J Appl Physiol 1986, 60(6):2040-2047.

46. Aubier M, Murciano D, Lecocguic $Y$, Viires $N$, Jacquens $Y$, Squara $P$, Pariente R: Effect of hypophosphatemia on diaphragmatic contractility in patients with acute respiratory failure. NEng/ J Med 1985, 313(7):420-424

47. Aubier M, Viires N, Piquet J, Murciano D, Blanchet F, Marty C, Gherardi R, Pariente R: Effects of hypocalcemia on diaphragmatic strength generation. J Appl Physiol 1985, 58(6):2054-2061

48. Dhingra S, Solven F, Wilson A, McCarthy DS: Hypomagnesemia and respiratory muscle power. Am Rev Respir Dis 1984, 129(3):497-498.

49. Watchko JF, LaFramboise WA, Standaert TA, Woodrum DE: Diaphragmatic function during hypoxemia: neonatal and developmental aspects. $J$ Appl Physiol 1986, 60(5):1599-1604

50. Watchko JF, Standaert TA, Woodrum DE: Diaphragmatic function during hypercapnia: neonatal and developmental aspects. J Appl Physio/ 1987, 62(2):768-775

51. Coirault C, Hagege A, Chemla D, Fratacci MD, Guerot C, Lecarpentier $Y$ : Angiotensin-converting enzyme inhibitor therapy improves respiratory muscle strength in patients with heart failure. Chest 2001, 119(6):1755-1760

52. Ribner HS, Sagar KB, Glasser SP, Hsieh AM, Dills CV, Larkin S, DeSilva J, Whalen JJ: Long-term therapy with benazepril in patients with congestive heart failure: effects on clinical status and exercise tolerance. J Clin Pharmacol 1990, 30(12):1106-1111.

53. Guazzi M, Palermo P, Pontone G, Susini F, Agostoni P: Synergistic efficacy of enalapril and losartan on exercise performance and oxygen consumption at peak exercise in congestive heart failure. Am J Cardiol 1999, 84(9):1038-1043

54. Onder $G$, Vedova $C D$, Pahor $M$ : Effects of $A C E$ inhibitors on skeletal muscle. Curr Pharm Des 2006, 12(16):2057-2064.

doi: 10.1186/1465-9921-11-57

Cite this article as: Dimitriou et al., Association of circulating angiotensin converting enzyme activity with respiratory muscle function in infants Respiratory Research 2010, 11:57 